\title{
Daily Cryptocurrency Returns Forecasting and Trading via Machine Learning
}

\author{
Andrew Falcon ${ }^{1}$ and Tianshu Lyu ${ }^{\#}$ \\ ${ }^{1}$ Phillips Academy, Andover, MA, USA \\ "Advisor
}

ABSTRACT

We execute a comparative analysis of machine learning models for the time-series forecasting of the sign of next-day cryptocurrency returns. We begin by compiling a proprietary dataset that encompasses a wide array of potential cryptocurrency valuation factors (price trends, liquidity, volatility, network, production, investor attention), subsequently identifying and evaluating the most significant factors. We apply eight machine learning models to the dataset, utilizing them as classifiers to predict the sign of next day price returns for the three largest cryptocurrencies by market capitalization: bitcoin, ethereum, and ripple. We show that the most significant valuation factors for cryptocurrency returns are price trend variables, seven and thirty-day reversal, to be specific. We conclude that support vector machines result in the most accurate classifications for all three cryptocurrencies. Additionally, we find that boosted models like AdaBoost and XGBoost have the poorest classification accuracy. At length, we construct a probabilitybased trading strategy that secures either a daily long or short position on one of the three examined cryptocurrencies. Ultimately, the strategy yields a Sharpe of 2.8 and a cumulative log return of 3.72. On average, the strategy's log returns outperformed standalone investments in all three cryptocurrencies by a factor of 5.64, and Sharpe ratios more than threefold.

\section{Introduction}

Cryptocurrency is a digital asset built on blockchain technology. It is a protocol where users can exchange digital assets in a decentralized manner - eliminating the need for a "middleman" - instead leaving transactions' verification to a peer-to-peer consensus enabled by cryptography. The three largest cryptocurrencies by market capitalization are bitcoin (BTC), ethereum (ETH), and ripple (XRP), respectively. Cryptocurrencies can be purchased in a myriad of ways, the most popular being a fiat currency exchange, wherein a user purchases a cryptocurrency with a fiat currency at the market's determined exchange rate. Recent fluctuations in the valuation of cryptocurrency prices and their subsequent returns has led investors, speculators, and academics alike to study the effectiveness of quantitative trading methods on this novel asset type.

Quantitative trading is a subject field where mathematical and computational tactics are utilized to construct predictive models and make inferences on the global financial markets. Many of the largest hedge funds and investment firms rely entirely or primarily on quantitative trading strategies to generate high return and/or low risk. Nevertheless, these strategies are typically applied to more traditional assets like equities and derivatives, for example. Although a wide array of research exists on the use of machine learning models to forecast the returns of traditional assets, significantly less exists on applying the same methodology to cryptocurrencies. With the recent rise in cryptocurrency's attention and valuation, we assess the effectiveness of quantitative trading strategies when applied to cryptocurrencies. More specifically, we utilize a total of eight machine learning classifiers to forecast the sign of next-day cryptocurrency price returns. The models consist of varying levels of complexity, evolving from simple logistic regressions to complex ensembles and boosted algorithms. We apply the research methodology to bitcoin, ethereum, and ripple. First, we analyze the variables utilized in the research, assessing the significance of their correlation 
towards returns. Second, we examine the classification accuracy of the tested models, and ultimately come to a conclusion on the most effective machine learning models, ranking them accordingly. Third, we develop a probabilitybased trading strategy that outperforms the returns and Sharpe ratios of a traditional long-only position in all three cryptocurrencies by an average factor of 5.64 and 3.15 , respectively.

\section{Related Work}

Early cryptocurrency research focuses primarily on bitcoin, since it is the first and largest cryptocurrency to date. More specifically, the academic debate revolved around two competing schools of thought - that is, whether bitcoin was to be classified as another currency or merely a speculative asset, with the bulk of scholars supporting the latter. The notion that cryptocurrency is a speculative asset with no underlying value has led numerous scholars to investigate the potential valuation factors of both price and returns. Kristoufek (2013), for example, shows a strong relationship between proxies for investor attention like Google and Wikipedia search trends and Bitcoin prices. Bouri et al. (2017) and Baur et al. (2018) demonstrate a weak correlation between bitcoin and financial variables like commodities, stocks, currencies, and bonds. Panagiotidis et al. (2019) compile a list of twenty-one factors of bitcoin price returns and also determine that Google Trends data is one of the most significant. Liu and Tsyvinski (2020) show that cryptocurrencies have no significant exposure to stock market, currencies, and macroeconomic factors. Conversely, they show that cryptocurrency-specific factors have a strong predictive power of returns. Moreover, they conclude that investor attention and time-series momentum are strong indicators of future cryptocurrency returns. The general consensus throughout the literature is that cryptocurrency-specific factors along with proxies for investor attention show the strongest correlation towards both prices and returns.

More recent research, however, has focused not solely on the analysis of cryptocurrency valuation factors, but also on the forecasting of future price returns, with the ultimate goal of developing a profitable trading strategy via machine learning techniques. Nevertheless, the effectiveness of these strategies has been polarizing. Jaquart, Dann, and Weinhardt (2021) assess the predictability of bitcoin across a relatively short time-series horizon (1 min to 60 min) and show that their long-short trading strategy generates negative returns after accounting for transaction fees. Sebastiao and Godinho (2021) develop a machine-learning trading strategy "with annualized Sharpe ratios of $80.17 \%$ and $91.35 \%$ and annualized returns (after proportional round-trip trading costs of $0.5 \%$ ) of $9.62 \%$ and $5.73 \%$, respectively". We build off the previous literature by assessing the significance of a myriad of cryptocurrency valuation factors, evaluating the effectiveness of machine learning classifiers for the sign prediction of next-day returns, and constructing a profitable probability-based trading strategy.

\section{Data}

\section{Basic Characteristics}

We collect data on six major valuation factors: Price trends, liquidity, volatility, network, production, and investor attention. Price trend factors consist of 7-day reversal, 30-day reversal, 6-month momentum, and 1-year momentum. Liquidity factors consist of 24-hour volume and market capitalization. Volatility factors consist of volatility as the standard deviation of returns (30-day, 90-day, and 180-day), beta, and beta squared. Network factors consist of transaction count, transfer count, active address count, and the median transaction fee (USD). Production factors consist of the average retail price of electricity in the United States for all sectors, the retail sales of electricity in the United States for all sectors, and the net generation of electricity in the United States for all sectors. Investor attention factors are comprised of the number of cryptocurrency wallet users and historical Google search trend queries. In essence, historical Google search trend queries are the number of weekly searches for the term "bitcoin". See Table 1 for an overview of the independent variables used within the research. 
Table 1. Input variables used for each cryptocurrency.

\begin{tabular}{|l|l|}
\hline Category & Variable \\
\hline Price Trends & Close price (USD) \\
\hline Price Trends & Log returns \\
\hline Price Trends & 7-day reversal \\
\hline Price Trends & 30-day reversal \\
\hline Price Trends & 6-month momentum \\
\hline Price Trends & 1-year momentum \\
\hline Liquidity & 24-hour volume \\
\hline Liquidity & 24-hour market cap \\
\hline Volatility & 30-day volatility \\
\hline Volatility & 90-day volatility \\
\hline Volatility & 180-day volatility \\
\hline Volatility & 90-day beta \\
\hline Volatility & 90-day beta squared \\
\hline Network & Median transaction fee (USD) \\
\hline Network & Active address count \\
\hline Network & Transaction count \\
\hline Network & Transfer count \\
\hline Investor attention & Number of wallet users \\
\hline Investor attention & Google search trend queries \\
\hline Production & Avg. retail price of electricity in the United States \\
\hline Production & Retail sales of electricity in the United States \\
\hline Production & Net generation of electricity in the United States \\
\hline
\end{tabular}

\section{Collection}

We collect data on close prices, volume, and market capitalization from coinmarketcap.com. Coinmarketcap.com notes that the close price of a cryptocurrency refers to the latest updated price for a given day. The data from coinmarketcap is utilized to construct all of the price trend and liquidity factors. Volatility variables are computed using close prices for BTC, ETH, and XRP, as well as cryptocurrency market close prices. We choose the Crescent Crypto Market Index (CCMIX) to represent cryptocurrency market returns. The CCMIX employs a market capitalization based weighted average to construct their index. We collect production data from the U.S. Energy Information Administration (EIA), and network data is collected from coinmetrics.io. Data on the number of wallet users is accessed from blockchain.com, and Google search trend queries are obtained from trends.google.com. We employ search data for the term "bitcoin", since it is larger, more liquid, and more widely recognized than any other cryptocurrency. Moreover, the term "bitcoin" is often used synonymously with the term "cryptocurrency" by those unfamiliar with blockchain technology. Overall, we visit trends.google.com, and download the historical data for the term "bitcoin" in the United States. The Google search trends are the historical datapoints of the weekly number of searches for the term "bitcoin".

\section{Variable Construction}

While a plethora of the research's independent variables are already processed and ready for use at the time of collection (e.g. production, investor attention, and network factors), we compute a handful of features manually. These 
include metrics for returns, volatility, beta, beta squared, momentum, and reversal. Additionally, our target variable, the sign of next-day returns, is constructed from our calculated log returns.

Equation 1: We construct the daily $\log$ return, $R_{d}$, at day $d$, where $P_{d}$ is the close price at day $d$ and $P_{d-1}$ is the close price at the day prior to day $d$ :

$$
R_{d}=\ln \left(\frac{P_{d}}{P_{d-1}}\right)
$$

Equation 2: We construct beta on a 90-day rolling window, where $c_{90}$ are the 90 daily $\log$ returns for cryptocurrency c. Conversely, $m_{90}$ are the 90 daily $\log$ returns for the cryptocurrency market, which we base on the CCMIX:

$$
\beta=\frac{\operatorname{cov}\left(c_{90}, m_{90}\right)}{\operatorname{var}\left(m_{90}\right)}
$$

Equation 3: Beta squared is constructed by squaring the respective beta calculation at a given day:

$$
(\beta)^{2}
$$

Equation 4: 30-day volatility, $V_{30}$, is constructed as the standard deviation of returns on a 30-day rolling window, where $c_{30}$ are the 30 daily $\log$ returns for cryptocurrency $c$ :

$$
V_{30}=\sqrt{\operatorname{var}\left(c_{30}\right)}
$$

Equation 5: 90-day volatility, $V_{90}$, is constructed as the standard deviation of returns on a 90-day rolling window, where $c_{90}$ are the 90 daily $\log$ returns for cryptocurrency $c$ :

$$
V_{90}=\sqrt{\operatorname{var}\left(c_{90}\right)}
$$

Equation 6: 180 -day volatility, $V_{180}$, is constructed as the standard deviation of returns on a 180-day rolling window, where $c_{180}$ are the 180 daily $\log$ returns for cryptocurrency $c$ :

$$
V_{180}=\sqrt{\operatorname{var}\left(c_{180}\right)}
$$

Equation 7: We calculate weekly reversal, $R e v_{7}$, as the 7-day holding period log return, where $P_{d}$ is the close price at current day $d$ and $P_{d-7}$ is the close price 7 days prior to day $d$ :

$$
R e v_{7}=\ln \left(\frac{P_{d}}{P_{d-7}}\right)
$$

Equation 8: We calculate monthly reversal, $\operatorname{Rev}_{30}$, as the 30-day holding period log return, where $P_{d}$ is the close price at current day $d$ and $P_{d-30}$ is the close price 30 days prior to day $d$ :

$$
R e v_{30}=\ln \left(\frac{P_{d}}{P_{d-30}}\right)
$$


Equation 9: We calculate 6-month momentum, $M_{6 m}$, where $P_{d-30}$ is the close price 30 days prior to current day $d$ and $P_{d-210}$ is the close price 210 days prior to current day $d$ :

$$
M_{6 m}=\ln \left(\frac{P_{d-30}}{P_{d-210}}\right)
$$

Equation 10: We calculate 1-year momentum, $M_{1 y}$, where $P_{d-30}$ is the close price 30 days prior to current day $d$ and $P_{d-395}$ is the close price 395 days prior to current day $d$ :

$$
M_{1 y}=\ln \left(\frac{P_{d-30}}{P_{d-395}}\right)
$$

Equation 11: Our target variable is the sign of daily log returns. We transform the returns into a binary classification problem, where 1 signifies a positive return and 0 represents a negative return. As such, the sign of daily returns at day $d, S_{d}$, is computed as follows:

$$
S_{d}= \begin{cases}1, & \text { if } R_{d+1}>0 \\ 0, & \text { otherwise }\end{cases}
$$

Note, since we are forecasting the sign of next-day returns, we shift our dataset such that the according sign value for day $d$ is the original sign of returns for day $d+1$.

\section{Cleaning Data}

Our independent variables exist in varying levels of time-series frequency. Data like close-price, market capitalization, and liquidity are available on a daily frequency. However, larger macroeconomic variables like the net generation, average retail price, and total retail sales of electricity are only available on a monthly frequency. Moreover, Google search trend data is available on a weekly frequency. Since our target variable is the next-day sign of returns, we transform all data into a daily frequency. To be more specific, if the data is in a monthly frequency, the single monthly value is matched for every day of the according month. In the scenario where data is in a weekly frequency, the single week value is matched for every value of that respective week. We begin our research with a time-sample from September $5^{\text {th }}, 2015$ to January $1^{\text {st }}, 2021$. However, since the construction of yearly momentum requires that data from the previous year be available, momentum data for the first year is unable to be calculated. Thus, we eliminate all data from 9/5/15 to 9/4/16. Our final dataset consists of a time-sample from September $5^{\text {th }}, 2016$ to January $1^{\text {st }}, 2021$.

\section{Variable Correlation}




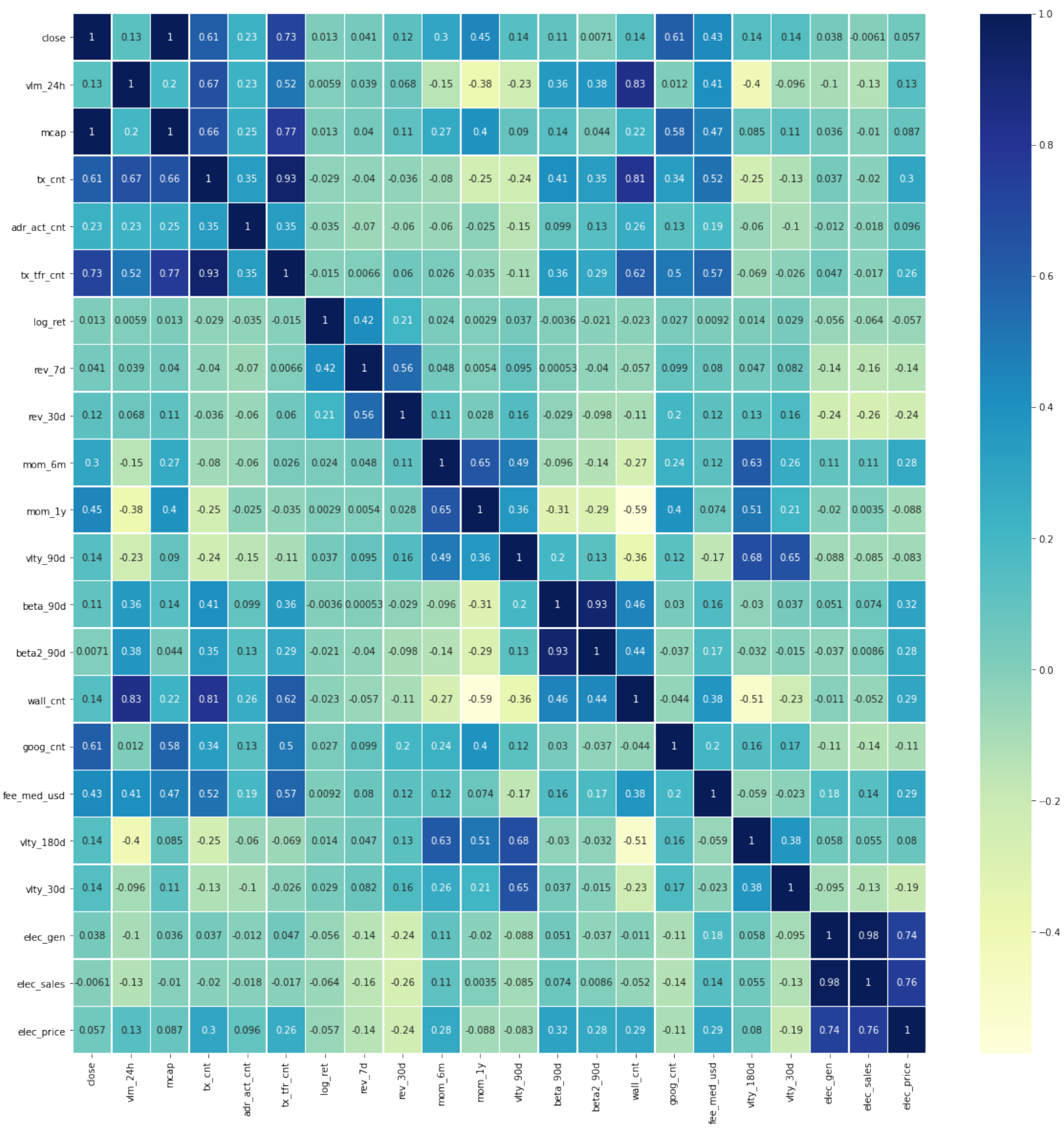

Figure 1. Heat map of all feature variable correlations. The displayed correlations are computed as the mean correlation value across BTC, ETH, and XRP for a given cell.

We reinforce the conclusion made by Liu and Tsyvinski (2020), substantiating that "there is a strong time-series momentum effect...". Additionally, of all variables analyzed within the research, the 7-day and 30-day reversals consistently maintain the strongest correlation towards returns. For all volatility variables, the 90 -day volatility showed the most significant correlation with returns. Regarding investor attention, Google search trend queries showed a stronger correlation than wallet count. Moreover, we find that production variables demonstrated a powerful, although negative, correlation with returns. Similarly, three out of the four examined network factors also showed a strong but negative correlation. Factors for liquidity, namely the 24-hour volume, were insignificant. 


\section{Methodology}

\section{Software Materials}

All data processing and analysis is done via Python 3.7. The dataset is cleaned and processed with Pandas and Numpy. Scikit-learn is utilized to implement the logistic regression, support vector machines, K-nearest neighbors, Gaussian process, decision tree, random forest, AdaBoost, and multilayer perceptron classifiers. The XGBoost is implemented via the native XGBoost Python library. Parameter optimization is done via autosklearn's Python package.

\section{Preprocessing}

We prepare our data for the machine learning models by using $80 \%$ training data and $20 \%$ testing data. Due to the time-series nature of our dataset, we avoid the use of cross-validation when training and testing data. Our feature variables are scaled to values between 0 and 1 using scikitlearn's MinMaxScaler. Overall, our dataset contained a target class imbalance, with the majority of all next-day targets having a positive sign. We combat the imbalanced data by applying a penalization score to the classes, utilizing scikitlearn's "class_weight" parameter to balance each class.

\section{Models}

We utilize a myriad of machine learning classification models to predict the sign of next-day returns. In all, our models included logistic regression, support vector machines, K-nearest neighbors, Gaussian process classifier, decision tree, random forest, AdaBoost, XGBoost, and multilayer perceptron. We apply the listed models to all three of our compiled cryptocurrency datasets: BTC, ETH, and XRP.

\section{Trading Strategy}

We develop a trading strategy that secures either a long or short position based not solely on the classification prediction, but also on the model's measured confidence that the classification is correct. In essence, we transform a mere binary classification problem into a probability prediction problem, where the model returns a probability for the sign of next day returns for the three cryptocurrencies. By optimizing our trading strategy to account for probability, we limit the positions that the model takes to solely those with the highest likelihood of classification success. Overall, we construct a simple probability-based trading strategy. First, for the three analyzed cryptocurrencies in this research (BTC, ETH, XRP), we predict the sign of next-day returns using what we determine to be the most accurate classification model for our dataset. Second, we take a single position each day on one cryptocurrency based on the model's prediction. The cryptocurrency we make a position on as well as the type of position (long or short) is the one with the model's highest probability of being correct. To be more specific, if the model determines that there is $55 \%$ probability for the next day sign of BTC to be positive, a 70\% probability for ETH to be negative, and a $60 \%$ probability for XRP to be positive, we take a short position on ETH. Positions are liquidated every 24 hours and a new position is taken based on the model's predictions for the next day's sign. Probabilities are determined using scikitlearn's “predict_proba" method. 


\section{Results}

\section{Models}

We find that the support vector machines give the most accurate classifications when forecasting the sign of next-day returns. On the contrary, we find that boosted methods like AdaBoost and XGBoost give the most inaccurate classifications. See Table 2 for a comprehensive overview of the various models' performance on each individual cryptocurrency.

Table 2. Classifiers and their accuracy for the next-day sign prediction of each cryptocurrency.

\begin{tabular}{|l|l|l|l|}
\hline Model & BTC Acc. & ETH Acc. & XRP Acc. \\
\hline Logistic regression & 0.5233 & 0.5374 & 0.5196 \\
\hline Support vector machines & 0.5698 & 0.5691 & 0.5572 \\
\hline K-nearest neighbors & 0.5172 & 0.5001 & 0.4973 \\
\hline Gaussian process & 0.5665 & 0.5686 & 0.5544 \\
\hline Decision tree & 0.5601 & 0.5624 & 0.5499 \\
\hline Random forest & 0.5665 & 0.5521 & 0.5501 \\
\hline AdaBoost & 0.4778 & 0.4851 & 0.4713 \\
\hline XGBoost & 0.5032 & 0.5005 & 0.4936 \\
\hline Multilayer perceptron & 0.5506 & 0.5442 & 0.5415 \\
\hline
\end{tabular}

\section{Trading Strategy}

We employ support vector machines for our long/short probability-based trading strategy, since our results show that it is the most accurate classifier for the sign prediction of next-day returns. See Table 3 for an overview of the cumulative log returns and Sharpe ratios for our trading strategy (SVM probability-based trading strategy), compared to the those from a standalone investment in BTC, ETH, or XRP over the same holding period.

Table 3. The research's trading strategy compared to a single long position in all three cryptocurrencies.

\begin{tabular}{|l|l|l|}
\hline Model & Log returns & Sharpe \\
\hline SVM probability-based trading strategy & 3.72 & 2.8 \\
\hline BTC & 1.11 & 1.49 \\
\hline ETH & 1.01 & 1.06 \\
\hline XRP & -0.15 & 0.13 \\
\hline
\end{tabular}

Overall, the SVM's probability-based trading strategy has a log return of 3.72. This correlates to a rate of return of approximately $41.3 \%$. Thus, with a $\$ 100$ investment, one would profit $\$ 41.3$.

\section{Discussion}

This research substantiates the literature's general consensus that machine learning classifiers are capable of predicting the next-day sign of returns for cryptocurrencies. Moreover, we corroborate the notion that both the returns and prices 
of cryptocurrencies maintain a strong relationship with time-series momentum. We find added significance in the use of additional price trend variables to forecast the next-day sign of returns, most notably with 7 and 30 -day reversal. Regarding the comparative analysis of machine learning models for the research's task, our study encompasses a larger list of models than the vast majority of work in the literature, eight total models, to be exact. Despite our larger array of models, they do lack diversity. More specifically, we utilized a substantial number of tree-based methods, quite possibly more than necessary. Additionally, the literature has found great success in the use of recurrent neural networks (RNN's), to which we used none. Despite the fundamental similarity shared across many of our models, this did pose some added benefits. When assessing the accuracy of the models, for example, we discovered that both XGBoost and AdaBoost showed the weakest classification accuracies. This further corroborated our conclusion that boosted methods have the least promise for the research's given prediction task. Had we instead used solely one boosted method; our conclusion would have been nowhere near as significant. We ultimately find that support vector machines demonstrate the highest classification accuracy for all three cryptocurrencies. We attribute the success of the support vector machines towards the dataset's relatively short time-series horizon, spanning a little over four years. Another significant observation from the research regards the predictability of ripple (XRP). We find that all models could more accurately forecast BTC and ETH than XRP. We suspect that this phenomenon could be attributed to XRP's more volatile nature.

Aside from assessing the significance of cryptocurrency valuation factors and machine learning models, the remainder of our research focused on developing a profitable trading strategy, to which we found great success. The majority of the research that exists on the forecasting of cryptocurrency returns, devises a trading strategy from only one cryptocurrency. By constructing our models such that they forecast the next-day sign of returns for BTC, ETH, and XRP, we are granted significantly more flexibility regarding the means by which we can construct a trading strategy. As such, we formulated one not solely by a model's predicted classification, but also the probabilities of the classification being correct for each of the analyzed cryptocurrencies.

Our trading strategy subsequently saw results that significantly outperformed standalone long positions in all three cryptocurrencies over the same holding period. The average log return of an investment in BTC, ETH, or XRP was 0.66 , whereas our support vector machines probability-based trading strategy showed a log return of 3.72. Additionally, the average Sharpe for the cryptocurrencies was 0.89. On the other hand, our strategy delivered a Sharpe of 2.8. Overall, the strategy formulated within this research outperformed log returns and Sharpe ratios by a factor of 5.64 and 3.15, respectively. Our strategy saw more success than studies focused on cryptocurrency predictability over short time horizons ( 1 and 60 minute). Taking a multitude of positions over small time periods makes a strategy more vulnerable to trading fees, which may seem marginal at first, but deteriorate returns over a longer period of time.

\section{Conclusion}

We capitalize on a wide array of machine learning models to continue the literature's study on the overall risks and returns of bitcoin (BTC), ethereum (ETH), and ripple (XRP). First, we begin our research by assessing the most significant valuation factors towards cryptocurrency prices and returns. Second, we execute a comparative analysis of machine learning models for the next-day sign prediction of the three cryptocurrencies. Third, we utilize our findings to develop a profitable, probability-based trading strategy.

We reinforce the conclusion made by Liu and Tsyvinski (2020), substantiating that "there is a strong timeseries momentum effect...". We also find that for all variables analyzed in the research, the 7 and 30 -day reversals show the strongest correlation towards returns. We conclude that support vector machines provide the highest classification accuracy when forecasting the sign of next day cryptocurrency returns. This is in contrast to boosted methods like AdaBoost and XGBoost, whose performance was the worst of the examined models. Ultimately, we employ our conclusions to construct a probability-based trading strategy that delivered a Sharpe of 2.8 and a cumulative log return of 3.72. In contrast, the according Sharpe ratios and returns were 1.11 and $1.49,1.01$ and 1.06 , and -0.15 and -0.13 for standalone long positions in BTC, ETH, and XRP, respectively. 


\section{Limitations}

Significant improvements could be made in future research to construct more accurate models and subsequently develop a more profitable trading strategy.

More specifically, a larger list of tested classifiers could potentially prove to be beneficial. The literature has shown success in cryptocurrency prediction via the use of recurrent neural networks, none of which were used in the research. Moreover, a more comprehensive parameter optimization for each of the tested models would likely result in positive contributions towards their accuracy. Building off the literature's conclusions that cryptocurrency returns have a significant relationship with momentum, our research could have also benefited from a deeper focus on varying frequencies of time-series data. Tests on different levels of time frequency like the previous 5 days, week, and month to predict the sign of next day returns may have improved the effectiveness of the models. A deeper emphasis on feature selection could have helped to eliminate unnecessary noise within the data, resulting in more accurate classifiers. In addition, the accuracy of the models could have been enhanced by compiling a dataset that consisted of more price trend related features, since our research shows that those variable categories posed the strongest predictive power. Regarding the trading strategy, we could have more accurately assessed its performance by testing the strategy in a wide array of changing market conditions, rather than the single market condition (bull) utilized within our testing data. Additionally, compiling a larger list of cryptocurrencies to the dataset may have resulted in both a more successful trading strategy, as well as a more comprehensive understanding of the variable correlations.

The most significant limitation we encountered throughout the research was in the data collection process. Due to the novelty of cryptocurrencies, limited time-series data is available for most variables. As a result, our training and testing data was of a considerably smaller size than datasets used for traditional assets like stocks and bonds. For the time being, however, little can be done to combat this issue. Over time, as cryptocurrency grows in attention as both a phenomenon and an investment, more data will become available, fostering the subsequent growth and advancement of literature.

\section{Acknowledgments}

We especially thank Tianshu Lyu from the Yale School of Management for his guidance, advice, and mentorship throughout the entirety of the research process. We also thank Lumiere Education for facilitating the relationship with Tianshu Lyu and for their general support in the formulation of the research paper.

\section{References}

Baur, D., Hong, K., \& Lee, A. (2018). Bitcoin: medium of exchange or speculative assets? Journal of International Financial Markets, Institutions and Money, 54, 177-189. https://doi.org/10.1016/j.intfin.2017.12.004

Bouri. E., Molnar. P., Azzi, G., Roubaud, D., \& Hagfors, L. (2017). On the hedge and safe haven properties of Bitcoin: Is it really more than a diversifier? Finance Research Letters, 20, 192-198. https://doi.org/10.1016/j.frl.2016.09.025

Gu, S., Kelly, B., \& Xiu, D. (2020). Empirical asset pricing via machine learning. Review of Financial Studies, 33(5), 2223-2273. https://doi.org/10.1093/rfs/hhaa009

Jaquart, P., Dann, D., \& Weinhardt, C. (2021). Short-term bitcoin market prediction via machine learning, Journal of Finance and Data Science, 7, 45-66. https://doi.org/10.1016/j.jfds.2021.03.001 
Kristoufek, L. (2013). Bitcoin meets Google trends and Wikipedia: quantifying the relationship between phenomena of the Internet era. Scientific Reports, 3, 3415. https://doi.org/10.1038/srep03415

Liu, Y., \& Tsyvinski, A. (2021). Risks and returns of cryptocurrency, Review of Financial Studies, 34(6), 26892727. https://doi.org/10.1093/rfs/hhaa113

Panagiotidis, T., Stengos, T., \& Vravosinos, O. (2019). The effects of markets, uncertainty and search intensity on bitcoin returns. International Review of Financial Analysis, 63, 220-242. https://doi.org/10.1016/j.irfa.2018.11.002

Sebastiao, H., \& Godinho, P. (2021). Forecasting and trading cryptocurrencies with machine learning under changing market conditions. Financial Innovation, 7, 3. https://doi.org/10.1186/s40854-020-00217-x 